PROCEEDINGS OF THE

AMERICAN MATHEMATICAL SOCIETY

Volume 129, Number 11, Pages 3379-3387

S 0002-9939(01)05952-4

Article electronically published on April 25, 2001

\title{
NON-TANGENTIAL LIMITS, FINE LIMITS AND THE DIRICHLET INTEGRAL
}

\author{
STEPHEN J. GARDINER
}

(Communicated by Albert Baernstein II)

\begin{abstract}
Let $B$ denote the unit ball in $\mathbb{R}^{n}$. This paper characterizes the subsets $E$ of $B$ with the property that $\sup _{E} h=\sup _{B} h$ for all harmonic functions $h$ on $B$ which have finite Dirichlet integral. It also examines, in the spirit of a celebrated paper of Brelot and Doob, the associated question of the connection between non-tangential and fine cluster sets of functions on $B$ at points of the boundary.
\end{abstract}

\section{INTRODUCTION}

Let $B(x, r)$ denote the open ball of centre $x$ and radius $r$ in Euclidean space $\mathbb{R}^{n}$ $(n \geq 2)$, and let $B=B(0,1)$. If $\mathcal{A}$ is a collection of harmonic functions on $B$, then it is natural to ask which non-empty subsets $E$ of $B$ have the property that

$$
\sup _{E} h=\sup _{B} h \text { for all } h \in \mathcal{A} \text {. }
$$

In the case where $\mathcal{A}=h^{\infty}$, the collection of all bounded harmonic functions on $B$, it is known (cf. [2]) that (11) holds if and only if $\sigma\left(E_{N T}\right)=\sigma(\partial B)$, where $\sigma$ denotes surface area measure on $\partial B$ and $E_{N T}$ is the (Borel) set of points of $\partial B$ which can be approached non-tangentially by a sequence in $E$. In the case where $\mathcal{A}=h^{1}$, the collection of differences of positive harmonic functions on $B$, it has been shown (see [10] and [8]) that (10) holds if and only if

$$
\int_{E(1 / 2)}|x-y|^{-n} d x=+\infty \text { for all } y \in \partial B,
$$

where $E(1 / 2)=\bigcup_{x \in E} B(x,(1-|x|) / 2)$. Below we present the corresponding result when $\mathcal{A}=\mathcal{D}$, the collection of all harmonic functions $h$ on $B$ which have finite Dirichlet integral; that is, $\int_{B}|\nabla h(x)|^{2} d x<+\infty$. We will use $\mathcal{C}(\cdot)$ to denote Newtonian (if $n \geq 3$ ) or logarithmic (if $n=2$ ) capacity on $\mathbb{R}^{n}$.

Theorem 1. Let $\emptyset \neq E \subseteq B$ and $\mathcal{A}=\mathcal{D}$. Then (1) holds if and only if $\mathcal{C}\left(E_{N T}\right)=$ $\mathcal{C}(\partial B)$.

When $n=2$, Theorem 1 is closely related to a recent result of Stray [13] concerning holomorphic functions in the Dirichlet space. However, our methods are completely different. If $\sigma\left(E_{N T}\right)=\sigma(\partial B)$, then it follows easily, by considering

Received by the editors December 17, 1999 and, in revised form, April 3, 2000.

2000 Mathematics Subject Classification. Primary 31B25.

(C)2001 American Mathematical Society 
Poisson integrals in $B$ of suitable potentials, that $\mathcal{C}\left(E_{N T}\right)=\mathcal{C}(\partial B)$. In fact, the capacitary condition is much weaker, as the following example shows.

Example 1. Let $n=2$, let $\mathbb{R}^{2}$ be identified with $\mathbb{C}$ in the usual manner, and let

$$
E=\left\{\left(1-2^{-2 j}\right) \exp \left(i k 2^{1-j} \pi\right): j \in \mathbb{N} \text { and } k=0,1, \ldots, 2^{j}-1\right\} .
$$

Then $\mathcal{C}\left(E_{N T}\right)=\mathcal{C}(\partial B)$, but $\sigma\left(E_{N T}\right)=0$. (See $\S 3.4$ for details.)

Brelot and Doob, in their landmark paper [3], were able to relate classical and potential theoretic boundary limit theorems by establishing the relationship between non-tangential and minimal fine cluster sets of functions. Inspired by their work and Theorem 1 we will now provide corresponding results which describe the relationship between non-tangential and fine cluster sets of functions.

Recall that the fine topology on $\mathbb{R}^{n}$ is the coarsest topology for which all superharmonic functions are continuous. A set $A$ is said to be thin at a point $x$ if $x$ is not a fine limit point of $A$. (For an account of these concepts see Chapter 1.XI of the book by Doob [7.) By Wiener's criterion, this is equivalent to the condition

$$
\sum_{k} 2^{k(n-2)} \mathcal{C}^{*}\left(\left\{y \in A: 2^{-k-1} \leq|x-y| \leq 2^{-k}\right\}\right)<+\infty \quad(n \geq 3)
$$

or

$$
\sum_{k} \frac{k}{\log 1 / \mathcal{C}^{*}\left(\left\{y \in A: 2^{-k-1} \leq|x-y| \leq 2^{-k}\right\}\right)}<+\infty \quad(n=2),
$$

where $\mathcal{C}^{*}(\cdot)$ denotes outer (Newtonian or logarithmic) capacity. If, instead, the weaker condition

$$
2^{k(n-2)} \mathcal{C}^{*}\left(\left\{y \in A: 2^{-k-1} \leq|x-y| \leq 2^{-k}\right\}\right) \rightarrow 0 \text { as } k \rightarrow \infty \quad(n \geq 3)
$$

or

$$
\frac{k}{\log 1 / \mathcal{C}^{*}\left(\left\{y \in A: 2^{-k-1} \leq|x-y| \leq 2^{-k}\right\}\right)} \rightarrow 0 \text { as } k \rightarrow \infty \quad(n=2)
$$

holds, then $A$ is said to be semi-thin at $x$. Now let $f: S \rightarrow[-\infty,+\infty]$, where $S \subseteq \mathbb{R}^{n}$, let $x \in \bar{S}$ and $l \in[-\infty,+\infty]$. We say that $l$ is a fine (respectively, semifine) cluster value of $f$ at $x$ if, for every neighbourhood $N$ of $l$ in $[-\infty,+\infty]$, the set $f^{-1}(N)$ is not thin (respectively, not semi-thin) at $x$. Finally, we define the non-tangential approach region

$$
K(z, \delta, \varepsilon)=\{x: \varepsilon>1-|x|>\delta|x-z|\}(z \in \partial B ; 0<\delta<1 ; 0<\varepsilon<1) .
$$

The following result is straightforward to prove, using ideas from [14].

Proposition 1. Let $h$ be a harmonic function on $K(z, \delta, \varepsilon)$ such that

$$
\int_{K(z, \delta, \varepsilon)}(1-|x|)^{2-n}|\nabla h(x)|^{2} d x<+\infty
$$

and let $\delta<\delta_{1}<1$. If there is a sequence $\left(x_{k}\right)$ of points in $K\left(z, \delta_{1}, \varepsilon\right)$ such that $x_{k} \rightarrow z$ and $h\left(x_{k}\right) \rightarrow l$, then $l$ is a semi-fine (and hence a fine) cluster value of $h$ at $z$. 
Less obvious is the next result, which goes in the opposite direction. If $f: B \rightarrow$ $[-\infty,+\infty]$, then the non-tangential and fine cluster sets of $f$ at a point $z \in \partial B$ are defined respectively by

$$
\begin{aligned}
C_{N T}(f, z)= & \left\{l \in[-\infty,+\infty]: f\left(x_{k}\right) \rightarrow l \text { for some sequence }\left(x_{k}\right)\right. \text { of } \\
& \text { points in } B \text { which approaches } z \text { non-tangentially }\}
\end{aligned}
$$

and

$$
C_{F}(f, z)=\{l \in[-\infty,+\infty]: l \text { is a fine cluster value of } f \text { at } z\} .
$$

Theorem 2. Let $f: B \rightarrow[-\infty,+\infty]$. Then there is a Euclidean- $G_{\delta}$ set $A \subseteq \partial B$ such that $\mathcal{C}(A)=\mathcal{C}(\partial B)$ and $C_{F}(f, z) \subseteq C_{N T}(f, z)$ whenever $z \in A$.

Theorem 2 can be viewed as a fine topology analogue of a maximality theorem of Collingwood and Lohwater (see Theorem 4.10 in [5]). We note that Mizuta [12] has also considered the relationship between non-tangential, normal and fine cluster sets. His results, which are specific to harmonic functions satisfying a Dirichlet-type integral condition, are of a completely different nature. The sharpness of Theorem 2 , even for harmonic functions, is demonstrated by the next result.

Theorem 3. Let $A \subseteq \partial B$ be a Euclidean- $G_{\delta}$ set such that $\mathcal{C}(A)=\mathcal{C}(\partial B)$. Then there is a harmonic function $h$ on $B$ such that $C_{F}(h, z)=[-\infty,+\infty]$ and $C_{N T}(h, z)$ $=\{0\}$ whenever $z \in \partial B \backslash A$.

Proposition 1 and Theorems 1 and 2 will be proved in $\S \S 2-4$ respectively. Theorem 3 will be proved in $\S 5$ using recent results concerning approximation by harmonic functions.

\section{Proof of Proposition 1}

Let $h$ be a harmonic function on $K(z, \delta, \varepsilon)$ such that

$$
\int_{K(z, \delta, \varepsilon)}(1-|x|)^{2-n}|\nabla h(x)|^{2} d x<+\infty,
$$

let $\delta<\delta_{0}<\delta_{1}<1$ and $0<\varepsilon_{1}<\varepsilon_{0}<\varepsilon$. Then there is a decreasing function $\phi:(0,+\infty) \rightarrow(0,+\infty)$ such that $\phi(t) \leq 2 \phi(2 t)$ for all $t$ and $\phi(t) \rightarrow+\infty$ as $t \rightarrow 0$, and such that $L<+\infty$, where

$$
L=\int_{K(z, \delta, \varepsilon)}(1-|x|)^{2-n} \phi(1-|x|)|\nabla h(x)|^{2} d x .
$$

Let $\eta_{0}>0$ be small enough so that $B\left(x, \eta_{0}(1-|x|)\right) \subseteq K(z, \delta, \varepsilon)$ whenever $x \in$ $K\left(z, \delta_{0}, \varepsilon_{0}\right)$. By the volume mean value inequality, applied to the subharmonic function $|\nabla h|^{2}$ and the ball $B\left(x, \eta_{0}(1-|x|)\right)$, we see that there is a positive constant $M$, depending only on $\eta_{0}$ and $n$, such that

$$
(1-|x|)^{2-n} \phi(1-|x|)|\nabla h(x)|^{2} \leq M L(1-|x|)^{-n} \quad\left(x \in K\left(z, \delta_{0}, \varepsilon_{0}\right)\right),
$$

and hence

$$
(1-|x|)|\nabla h(x)| \leq \sqrt{\frac{M L}{\phi(1-|x|)}} \quad\left(x \in K\left(z, \delta_{0}, \varepsilon_{0}\right)\right) .
$$

Let $\eta_{1}>0$ be small enough so that $B\left(x, \eta_{1}(1-|x|)\right) \subseteq K\left(z, \delta_{0}, \varepsilon_{0}\right)$ whenever $x \in K\left(z, \delta_{1}, \varepsilon_{1}\right)$. Also, let $\left(x_{k}\right)$ be a sequence of points in $K\left(z, \delta_{1}, \varepsilon_{1}\right)$ such that 
$x_{k} \rightarrow z$ and $h\left(x_{k}\right) \rightarrow l$, and let $D=\bigcup_{k} B\left(x_{k}, \eta_{1}\left(1-\left|x_{k}\right|\right)\right)$. It follows from (2) and the mean value theorem of differential calculus that

$$
h(x) \rightarrow l \quad(x \rightarrow z ; x \in D),
$$

in view of the fact that $\phi(t) \rightarrow+\infty$ as $t \rightarrow 0$. Since $\mathcal{C}(B(x, r))$ is $r^{n-2}(n \geq 3)$ or $r$ $(n=2)$, it is clear that $D$ is not semi-thin at $z$. Thus $l$ is a semi-fine (and hence a fine) cluster value of $h$ at $z$.

\section{Proofs of Theorem 1 and Example 1}

3.1. For the proofs of Theorems $1-3$ we will assume that $n \geq 3$ and omit the minor modifications required to adapt our arguments to the plane. Before proving Theorem 1 we will assemble some preliminary observations. A function $f: S \rightarrow$ $[-\infty,+\infty]$ is said to have a fine limit at a fine limit point $x$ of $S$ if $C_{F}(f, x)$ consists of only one point. If, further, $C_{F}(f, x)=\{f(x)\}$, then $f$ is said to be finely continuous at $x$.

Lemma A. Let $f: B \rightarrow[0,+\infty)$ be integrable on $B$ and let $0<\varepsilon<1$. Then there is a set $Y \subset \partial B$, of zero $(n-2)$-dimensional Hausdorff measure, such that

$$
\int_{K(z, \delta, \varepsilon)}(1-|x|)^{2-n} f(x) d x<+\infty \quad(z \in \partial B \backslash Y ; 0<\delta<1) .
$$

Theorem A. If $h \in \mathcal{D}$, then there is a finite-valued extension $\bar{h}$ of $h$ to $\bar{B}$, and a polar set $Z \subset \partial B$, such that

$$
h(r z) \rightarrow \bar{h}(z) \quad(r \rightarrow 1-; \quad z \in \partial B \backslash Z)
$$

and $\bar{h}$ is finely continuous at each point of $\partial B \backslash Z$.

In proving Lemma $\mathrm{A}$ it is clearly enough to show that, for a fixed choice of $\delta$, there is a set $Y_{\delta} \subset \partial B$, of zero $(n-2)$-dimensional Hausdorff measure, such that

$$
\int_{K(z, \delta, \varepsilon)}(1-|x|)^{2-n} f(x) d x<+\infty \quad\left(z \in \partial B \backslash Y_{\delta}\right) .
$$

This can be done by imitating the proof of the analogous result for functions on half-spaces, which may be found in Lemma 5 of [14].

Theorem A is taken from Deny ([6], Chap. IV, Théorème 3), who proved the result for the more general class of Beppo Levi functions $h$ on $B$. Actually, Deny's result asserts only that $\bar{h}$ has a fine limit at each point of $\partial B \backslash Z$, but the proof makes it clear that $\bar{h}$ is actually finely continuous at each point of a set which has this form.

Lemma 1. The unit sphere $\partial B$, with the topology induced on it by the fine topology on $\mathbb{R}^{n}$, is a Baire space.

To prove Lemma 1 , we note that the fine topology on $\mathbb{R}^{n}$ has a base which consists of (Euclidean) compact sets. The same is therefore also true of the topology it induces on $\partial B$. We can now adopt the argument given on pp. 167, 168 of [7] to see that this space is Baire.

If $A$ is a bounded set in $\mathbb{R}^{n}$, then we use $\widehat{R}_{1}^{A}$ to denote the capacitary potential of $A$. Thus the Riesz measure $\nu_{A}$ associated with $\widehat{R}_{1}^{A}$ satisfies $\nu_{A}\left(\mathbb{R}^{n}\right)=\mathcal{C}^{*}(A)$.

Lemma 2. Let $A \subseteq \partial B$ and $z \in \partial B$. If $A$ is thin at $z$, then $\widehat{R}_{1}^{A}(z)<1$. 
To see this we note that, if $z \in \bar{A}$, then there is a positive superharmonic function $u$ on $\mathbb{R}^{n}$ such that

$$
\liminf _{x \rightarrow z, x \in A} u(x)>u(z) .
$$

If we define $v(x)=u(x)+a|x+z|^{2-n}$, where $a$ is a suitably chosen positive number, then $v$ is a positive superharmonic function on $\mathbb{R}^{n}$ such that

$$
\inf _{A \backslash\{z\}} v>v(z) .
$$

It follows easily that $\widehat{R}_{1}^{A}(z)<1$, and this inequality is obviously also true when $z \notin \bar{A}$.

3.2. We now turn to the "if" part of Theorem 1. Suppose that $\mathcal{C}\left(E_{N T}\right)=\mathcal{C}(\partial B)$ and suppose further, for the sake of contradiction, that $E_{N T}$ is thin at some point $z$ of $\partial B$. Then $\widehat{R}_{1}^{E_{N T}}(z)<1$, by Lemma 2 , and this leads to the contradictory conclusion that $\mathcal{C}\left(E_{N T}\right)=\widehat{R}_{1}^{E_{N T}}(0)<1=\mathcal{C}(\partial B)$. Hence the fine closure of $E_{N T}$ is all of $\partial B$.

Now let $h \in \mathcal{D}$. By Theorem A, there exist a function $\bar{h}: \bar{B} \rightarrow \mathbb{R}$ and a polar set $Z \subset \partial B$ such that $\left.\bar{h}\right|_{B}=h$ and (3) holds, and such that $\bar{h}$ is finely continuous at all points of $\partial B \backslash Z$. Let $M=\sup _{E} h$ and suppose, to avoid triviality, that $M<+\infty$. By Proposition 1 and Lemma A (with $f=|\nabla h|^{2}$ ), there is a polar subset $Y$ of $\partial B$ such that $\bar{h} \leq M$ on $E_{N T} \backslash(Y \cup Z)$. (Here we are using the fact that a bounded set of finite $(n-2)$-dimensional Hausdorff measure is polar; see [4], Chap. IV, Theorem $1)$. The fine closure of $E_{N T} \backslash(Y \cup Z)$ is also $\partial B$, so $\bar{h} \leq M$ on $\partial B \backslash Z$. We note that the subharmonic function $h^{2}$ has a harmonic majorant on $B$ because

$$
\int_{B}(1-|x|)\left(\Delta\left(h^{2}\right)\right)(x) d x \leq 2 \int_{B}|\nabla h(x)|^{2} d x<+\infty .
$$

Hence $h$ is equal to the Poisson integral of a function $g$ on $\partial B$ (see [7, 1.II.14). Further, from (3) and Fatou's boundary limit theorem, $g=\left.\bar{h}\right|_{\partial B}$ almost everywhere $(\sigma)$ on $\partial B$, and so $h \leq M$ on $B$. Thus (I) holds and we have now established the "if" part of Theorem 1.

3.3. Conversely, suppose that (11) holds and let

$$
F(r)=\partial B \cap\left(\bigcup_{x \in E \backslash B(0, r)} B(x, 2(1-|x|))\right) \quad(0<r<1) .
$$

Clearly (1) implies that $\bar{E} \cap \partial B \neq \emptyset$, so $F(r) \neq \emptyset$ for all $r$. Suppose further, for the sake of contradiction, that there exists $r$ such that the fine closure of $F(r)$ is a proper subset of $\partial B$. Then the function $h=1-\widehat{R}_{1}^{F(r)}$, which is harmonic on $B$, is strictly positive there, in view of Lemma 2 . We note (see [7], 1.IV.5) that there is an increasing sequence $\left(u_{k}\right)$ of $C^{\infty}$ Newtonian potentials, with associated measures $\left(\mu_{k}\right)$, such that $u_{k} \uparrow \widehat{R}_{1}^{F(r)}$ and $u_{k}$ is harmonic outside $\overline{B(0,1+1 / k)} \backslash B(0,1-1 / k)$ and that

$$
\int_{\mathbb{R}^{n}}\left|\nabla u_{k}(x)\right|^{2} d x=a_{n} \int u_{k} d \mu_{k}
$$


by Green's first identity, where $a_{n}$ is a positive constant depending only on $n$. Since $\left|\nabla u_{k}\right| \rightarrow|\nabla h|$ locally uniformly on $B$, it follows from the reciprocity law that

$$
\int_{B}|\nabla h(x)|^{2} d x \leq a_{n} \int \widehat{R}_{1}^{F(r)} d \nu_{F(r)} \leq a_{n} \mathcal{C}(F(r))<+\infty
$$

that is, $\left.h\right|_{B} \in \mathcal{D}$.

Let $M=\sup _{B} h$ and let $v_{r}$ denote the harmonic measure of $\partial B \backslash F(r)$ in $B$. The definition of $F(r)$ ensures that there is a constant $c \in(0,1)$, independent of $E$, such that $v_{r} \leq c$ on $E \backslash B(0, r)$. Since $h=1-\widehat{R}_{1}^{F(r)}=0$ on $F(r)$, it follows that $h \leq c M$ on $E \backslash B(0, r)$, and so

$$
\sup _{E} h \leq \max \left\{c M, \sup _{B(0, r)} h\right\}<M=\sup _{B} h .
$$

This contradicts (1). Hence, for every $r \in(0,1)$, the fine closure of $F(r)$ is all of $\partial B$.

Let

$$
F=\bigcap_{k=1}^{\infty} F\left(\frac{k}{k+1}\right) .
$$

Then, by Lemma 1 , the fine closure of $F$ is also all of $\partial B$. Finally, it is clear that $F \subseteq E_{N T}$, so the fine closure of $E_{N T}$ is also $\partial B$. Thus $\hat{R}_{1}^{E_{N T}}=\hat{R}_{1}^{\partial B}$, and hence $\mathcal{C}\left(E_{N T}\right)=\mathcal{C}(\partial B)$, as required.

3.4. We now present the details of Example 1. Let $E$ be the set defined there. Then

$$
E_{N T}=\bigcup_{l=1}^{\infty} \bigcap_{m=1}^{\infty} \bigcup_{j=m}^{\infty} \bigcup_{k=0}^{2^{j}-1} F_{j, k, l}
$$

where

$$
F_{j, k, l}=\left\{e^{i \theta}:\left|\theta-k 2^{1-j} \pi\right|<l 2^{-2 j}\right\} .
$$

The set $F_{j, k, l}$ is an open arc of the unit circle and $\mathcal{C}\left(F_{j, k, l}\right) \geq l 2^{-2 j-2}$ (see [11, p. $173,(2.4 .4))$. It follows easily from Wiener's criterion that the set

$$
A_{l, m}=\bigcup_{j=m}^{\infty} \bigcup_{k=0}^{2^{j}-1} F_{j, k, l}
$$

is finely dense in the circle. Hence, for any $l \in \mathbb{N}$, the set $\bigcap_{m=1}^{\infty} A_{l, m}$ is finely dense in the circle, by Lemma 1. It follows that $\mathcal{C}\left(E_{N T}\right)=\mathcal{C}(\partial B)$. However,

$$
\sigma\left(A_{l, m}\right) \leq \sum_{j=m}^{\infty} \sum_{k=0}^{2^{j}-1} l 2^{1-2 j}=l 2^{2-m}
$$

so $\sigma\left(\bigcap_{m=1}^{\infty} A_{l, m}\right)=0$ and hence $\sigma\left(E_{N T}\right)=0$, as claimed. 


\section{Proof of Theorem 2}

Let $f: B \rightarrow[-\infty,+\infty]$ and

$$
D=\left\{z \in \partial B: C_{F}(f, z) \backslash C_{K}(f, z) \neq \emptyset\right\},
$$

where, for each $z \in \partial B$,

$$
\begin{aligned}
C_{K}(f, z)= & \left\{l \in[-\infty,+\infty]: f\left(x_{k}\right) \rightarrow l \text { for some sequence }\left(x_{k}\right)\right. \text { of } \\
& \text { points in } \left.K(z, 1 / 2,1 / 2) \text { such that } x_{k} \rightarrow z\right\} .
\end{aligned}
$$

Further, let $\mathcal{I}$ denote the collection of closed intervals of $[-\infty,+\infty]$ with endpoints in $\mathbb{Q} \cup\{-\infty,+\infty\}$. Suppose that $z \in D$. Since $C_{K}(f, z)$ is a compact subset of $[-\infty,+\infty]$, we can find $I \in \mathcal{I}$, a finite union $J$ of intervals from $\mathcal{I}$, and $\varepsilon \in \mathbb{Q} \cap(0,1)$ such that

$$
I \cap C_{F}(f, z) \neq \emptyset, \quad I \cap J=\emptyset
$$

and

$$
f\left(K\left(z, \frac{1}{2}, \varepsilon\right)\right) \subseteq J .
$$

If $I, J$ and $\varepsilon$ are as above, then we say that $z \in D(I, J, \varepsilon)$. Thus

$$
D \subseteq \bigcup_{I, J, \varepsilon} D(I, J, \varepsilon)
$$

where the union is over all possible choices of $I, J, \varepsilon$.

Now suppose that one of the sets in this union, $D_{0}=D\left(I_{0}, J_{0}, \varepsilon_{0}\right)$ say, has the property that its (Euclidean) closure has non-empty interior $U$ with respect to the fine-induced topology on $\partial B$, let

$$
E=B \backslash\left(\bigcup_{z \in D_{0}} K\left(z, \frac{1}{2}, \varepsilon_{0}\right)\right)
$$

and $h=1-\widehat{R}_{1}^{\partial B \backslash U}$. Then $h=0$ on $\partial B \backslash U$ since the latter set is finely perfect. Also, $\bar{E} \cap \partial B \neq \emptyset$ by (4) and (5), and

$$
B(x, 2(1-|x|)) \cap U=\emptyset \quad\left(x \in E \backslash \overline{B\left(0,1-\varepsilon_{0}\right)}\right)
$$

by the definition of $E$. Reasoning as in $\S 3.3$, we now see that $h>0$ on $B$, and on $U$ by Lemma 2, but $\sup _{E} h<\sup _{B} h$. Let $\sup _{E} h<M<\sup _{B} h$ and $V=$ $\left\{x \in \mathbb{R}^{n}: h(x)>M\right\}$, and choose $z_{1} \in U$ such that $h\left(z_{1}\right)>M$. Then $V$ is a fine neighbourhood of $z_{1}$ and $V \cap E=\emptyset$. It now follows from (5) and (7) that $C_{F}\left(f, z_{1}\right) \subseteq J_{0}$, but this contradicts (4). Thus $U=\emptyset$.

Let

$$
A=\bigcap_{I, J, \varepsilon}(\partial B \backslash \overline{D(I, J, \varepsilon)}) .
$$

Thus $A$ is a (Euclidean) $G_{\delta}$-set and each of the sets $\partial B \backslash \overline{D(I, J, \varepsilon)}$ is open and dense in the fine-induced topology on $\partial B$. By Lemma 1, the fine closure of $A$ is $\partial B$ and hence $\mathcal{C}(A)=\mathcal{C}(\partial B)$. Since $C_{K}(f, z) \subseteq C_{N T}(f, z)$, it follows that

$$
C_{F}(f, z) \subseteq C_{N T}(f, z) \quad(z \in \partial B \backslash D) .
$$

From ([6) and (8) we see that $A \subseteq \partial B \backslash D$, and so Theorem 2 is established. 


\section{Proof of Theorem 3}

Let $A \subseteq \partial B$ be a Euclidean- $G_{\delta}$ set such that $\mathcal{C}(A)=\mathcal{C}(\partial B)$. As we argued at the beginning of $\S 3.2$, it follows that $A$ is finely dense in $\partial B$. Thus there is an increasing sequence $\left(F_{k}\right)$ of compact sets such that $\partial B \backslash A=\bigcup_{k} F_{k}$ and such that $\partial B \backslash F_{k}$ is finely dense in $\partial B$ for each $k$. To avoid trivialities we may assume that the sets $\partial B \backslash A$ and $F_{1}$ are non-empty.

Let $g_{k}: \partial B \rightarrow[0,1)$ be defined by

$$
g_{k}(z)=\frac{\left\{\operatorname{dist}\left(z, F_{k}\right)\right\}^{2}}{5} \quad(z \in \partial B ; k \in \mathbb{N}) .
$$

Then the sets

$$
\begin{gathered}
E_{k}=\left\{r z: z \in \partial B \backslash F_{k} \text { and } 1-r=\frac{g_{k}(z)}{3 k-1}\right\}(k \in \mathbb{N}), \\
D_{1}=B \cap\left\{r z: z \in \partial B \text { and } 1-r \geq g_{1}(z)\right\},
\end{gathered}
$$

and

$$
D_{k+1}=B \cap\left\{r z: z \in \partial B \text { and } \frac{g_{k}(z)}{3 k} \geq 1-r \geq \frac{g_{k+1}(z)}{3 k+1}\right\} \quad(k \in \mathbb{N})
$$

are all closed relative to $B$ and are pairwise disjoint. Let

$$
E=\left(\bigcup_{k} E_{k}\right) \cup\left(\bigcup_{k} D_{k}\right)
$$

and let $B^{*}$ denote the Alexandroff (one-point) compactification of $B$. Since, for a given value of $r$ in $(0,1)$, only finitely many sets in the union in (9) meet $B(0, r)$, the set $E$ is also closed relative to $B$.

If we define $u: E \rightarrow \mathbb{R}$ by

$$
u(x)=\left\{\begin{aligned}
0 & \text { if } \quad x \in \bigcup_{k} D_{k}, \\
q_{k} & \text { if } \quad x \in E_{k} ; k \geq 1,
\end{aligned}\right.
$$

where $\left(q_{k}\right)$ is an enumeration of $\mathbb{Q}$, then $u$ extends to a locally constant (and hence harmonic) function on an open set which contains $E$. It is easy to check that $B^{*} \backslash E$ is connected and locally connected (see $\S 3.2$ of [9] for a discussion of local connectedness in this context). We note that, if $z \in \partial B \backslash A$ and $0<\delta<1$, then there exists a (smallest) number $k_{0}$ such that $z \in F_{k_{0}}$ and a number $\varepsilon_{z, \delta}$ in $(0,1)$ such that

$$
K\left(z, \delta, \varepsilon_{z, \delta}\right) \subseteq D_{k_{0}} \subseteq \bigcup_{k} D_{k} \quad(0<\delta<1)
$$

We also claim that, if $z \in \partial B \backslash A$, then $E_{k}$ is non-thin at $z$ for all sufficiently large $k$. To see this, we choose $k_{0}$ such that $z \in F_{k_{0}}$ and suppose that $E_{k}$ is thin at $z$ for some $k \geq k_{0}$. Since the radial projection map from $E_{k}$ to $\partial B \backslash F_{k}$ is a Lipschitz map with Lipschitz constant 2, we have

$$
\begin{aligned}
& \mathcal{C}\left(\left\{y \in \partial B \backslash F_{k}: 2^{-j-1} \leq|y-z| \leq 2^{-j}\right\}\right) \\
\leq \quad & 2^{n-2} \mathcal{C}\left(\left\{y \in E_{k}: 2^{-j-2} \leq|y-z| \leq 2^{-j+1}\right\}\right)
\end{aligned}
$$

for each $j \in \mathbb{N}$, by standard contraction and dilation properties of Newtonian capacity (see [11], Chap. 2, §3). Hence, by Wiener's criterion, we obtain the contradictory conclusion that $\partial B \backslash F_{k}$ is also thin at $z$. Thus our claim is verified. 
We now apply a recent harmonic approximation result (see [1], or Corollary 3.10 in [9]) to observe that there is a harmonic function $h$ on $B$ such that

$$
|h(x)-u(x)|<1-|x| \quad(x \in E) .
$$

It follows from (10) and (11) that $C_{N T}(h, z)=\{0\}$ whenever $z \in \partial B \backslash A$. For such $z$, it also follows from (10) and the claim verified in the previous paragraph that $C_{F}(h, z)$ contains all but a finite number of the rationals, and so $C_{F}(h, z)=$ $[-\infty,+\infty]$. The proof of Theorem 3 is now complete.

\section{REFERENCES}

1. D. H. Armitage and M. Goldstein, Tangential harmonic approximation on relatively closed sets, Proc. London Math. Soc. (3), 68 (1994), 112-126. MR 94i:31005

2. F. F. Bonsall, Domination of the supremum of a bounded harmonic function by its supremum over a countable subset, Proc. Edinburgh Math. Soc., 30 (1987), 471-477. MR 88k:31001

3. M. Brelot and J. L. Doob, Limites angulaires et limites fines, Ann. Inst. Fourier Grenoble, 13 (1963), 395-415. MR 33:4299

4. L. Carleson, Selected problems on exceptional sets, Van Nostrand, Princeton, 1967. MR 37:1576

5. E. F. Collingwood and A. J. Lohwater, The theory of cluster sets, Cambridge Univ. Press, Cambridge, 1966. MR 38:325

6. J. Deny, Les potentiels d'énergie finie, Acta Math. 82 (1950), 107-183. MR 12:98e

7. J. L. Doob, Classical potential theory and its probabilistic counterpart, Springer, New York, 1983. MR 85k:31001

8. S. J. Gardiner, Sets of determination for harmonic functions, Trans. Amer. Math. Soc. 338 (1993), 233-243. MR 93j:31005

9. S. J. Gardiner, Harmonic approximation, London Math. Soc. Lecture Note Series 221, Cambridge Univ. Press, Cambridge, 1995. MR 96j:31001

10. W. K. Hayman and T. J. Lyons, Bases for positive continuous functions, J. London Math. Soc. (2) 42 (1990), 292-308. MR 92a:31002

11. N. S. Landkof, Foundations of modern potential theory, Springer, Berlin, 1972. MR 50:2520

12. Y. Mizuta, On the behaviour of harmonic functions near a hyperplane, Analysis 2 (1982), 203-218.

13. A. Stray, Simultaneous approximation in the Dirichlet space, in: Advances in Multivariate Approximation, ed. W. Haussmann et al., Wiley, Berlin, 1999, pp. 307-319.

14. H. Wallin, On the existence of boundary values of a class of Beppo Levi functions, Trans. Amer. Math. Soc. 120 (1965), 510-525. MR 32:5911

Department of Mathematics, University College Dublin, Dublin 4, Ireland

E-mail address: stephen.gardiner@ucd.ie 